\title{
Enhanced Plasticity in Zincergic, Cortical Circuits after Exposure to Enriched Environments
}

\author{
Amy S. Nakashima ${ }^{1,2}$ and Richard H. Dyck ${ }^{1,2,3}$ \\ ${ }^{1}$ Department of Psychology, ${ }^{2}$ Hotchkiss Brain Institute, and ${ }^{3}$ Department of Cell Biology and Anatomy, University of Calgary, Calgary, Alberta, \\ Canada T2N $1 \mathrm{~N} 4$
}

\begin{abstract}
Despite the plethora of reports that demonstrate plasticity in the mammalian cerebral cortex, the characterization of the cellular mechanisms that mediate it is sparse. Here, we show that the magnitude of the experience-dependent regulation of vesicular zinc is significantly increased through enriched-environment housing. Mice were reared either in a deprived environment and subsequently housed in deprived, minimally enriched, or enriched conditions after the removal of the c-row of vibrissae or reared in an enriched environment before and after vibrissae removal. Levels of vesicular zinc were assessed in deprived and nondeprived barrels $6 \mathrm{~h}$ to $14 \mathrm{~d}$ after vibrissae removal. We found that housing in enriched environmental conditions resulted in a greater change in vesicular zinc levels than did other housing conditions; however, this effect was dependent on both the magnitude and duration of enrichment. Our data indicate that enriched-environment housing has profound effects on the regulation of vesicular zinc that occurs concurrently with experiencedependent plasticity, suggesting a role for zinc in the multitude of cortical modifications associated with enriched environments.
\end{abstract}

Key words: zinc; barrel cortex; cortical plasticity; enrichment; somatosensory cortex; synaptic plasticity

\section{Introduction}

The divalent cation zinc is an essential element, having a multitude of general and specialized functions in the body. In the brain, after transport across the blood-brain barrier, the majority of zinc is bound to proteins having regulatory and structural functions. However, $15-20 \%$ of zinc in the brain is found within synaptic vesicles in a subset of glutamatergic neurons (Frederickson, 1989; Beaulieu et al., 1992) and is released in a voltage- and activity-dependent manner (Assaf and Chung, 1984; Howell et al., 1984). Zinc can potently alter the functioning of a variety of neurotransmitter receptors, as well as modulate second messenger systems (Westbrook and Mayer, 1987; Paoletti et al., 1997; Huang et al., 2008). Furthermore, the experimental induction of plasticity, long-term potentiation and depression (LTP and LTD, respectively), has been shown to be robustly affected by zinc concentrations (Li et al., 2001; Izumi et al., 2006; Huang et al., 2008).

Conversely, experience-dependent plasticity produced through the manipulation of sensory input is accompanied by changes in vesicular zinc levels. For example, the removal or stimulation of vibrissae is a well characterized method that results in considerable plasticity within the barrels of the somatosensory cortex (Glazewski and Fox, 1996; Bender et al., 2006). Such manipulations have been shown to result in a rapid increase or decrease (with vibrissae removal or stimulation, respectively) in vesicular zinc levels within the corresponding barrels (Czupryn

Received Sept. 28, 2008; revised 0ct. 28, 2008; accepted Nov. 14, 2008.

This work was supported by the Natural Sciences and Engineering and Research Council of Canada in the form of a Discovery grant (R.H.D.) and graduate scholarship (A.S.N.).

Correspondence should be addressed to Dr. Richard H. Dyck, Department of Psychology, University of Calgary, 2500 University Drive NW, Calgary, Alberta, Canada T2N 1N4. E-mail: rdyck@ucalgary.ca.

DOI:10.1523/JNEUROSCI.4645-08.2008

Copyright $\odot 2008$ Society for Neuroscience ～0270-6474/08/2813995-05\$15.00/0 and Skangiel-Kramska, 2001b; Brown and Dyck, 2002, 2005). Changes in vesicular zinc levels that occur concurrently with experience-dependent plasticity, together with the regulatory effects of zinc on neurotransmission, are suggestive of a modulatory effect of zinc on this type of cortical plasticity.

To expand on these findings, we have examined how exposure to enriched environments altered the dynamics of vesicular zinc regulation in experience-dependent plasticity generated by vibrissae plucking. Housing laboratory animals in enriched environments that promote sensory, motor, and social stimulation has robust effects on the structure and function of the cortex. For instance, enriched environmental housing promotes improvements in behavioral tasks (Venable et al., 1988; Leggio et al., 2005), leads to alterations in the morphology and physiology of cortical neurons, and, in certain brain regions, increases neurogenesis (Sirevaag and Greenough, 1988; Kempermann et al., 1997; Leggio et al., 2005). Electrophysiologically, enriched environments can induce fundamental alterations in the induction and maintenance of LTP/LTD (Foster et al., 1996; Foster and Dumas, 2001; Abraham et al., 2002; Artola et al., 2006; Irvine et al., 2006). Thus, housing in enriched conditions results in a multitude of cortical changes, some of which may involve a zincergic component.

Utilizing different magnitudes and durations of enrichment, we examined how enriched environments affected the experience-dependent regulation of vesicular zinc after vibrissae removal. Our results demonstrate that a greater increase in vesicular zinc staining is observed within barrels deprived of sensory input when mice are housed in enriched conditions, indicating that changes in vesicular zinc regulation may contribute to the changes in plasticity that occur with enriched-environment housing. 


\section{Materials and Methods}

All mice were provided food and water ad libitum and were housed in standard laboratory housing under a $12 \mathrm{~h}$ light/dark cycle for the duration of the experiment. All procedures were authorized by the Animal Care Committee of the University of Calgary and mice were handled in accordance with the guidelines dictated by the Canadian Council for Animal Care.

In total, four groups of different environmental manipulations were used to examine the effect of enriched housing conditions on the regulation of vesicular zinc after the vibrissae plucking (Fig. 1). These groups were chosen to delineate the effect of differing degrees of enrichment on the vesicular zinc response. The first three groups examined the effect of environment after the removal of vibrissae. In these groups, male C57BL/6 mice were housed in a deprived environmental condition after weaning, consisting of a standard shoebox cage $(13 \times 17.5 \times 28 \mathrm{~cm})$ with mice housed two to a cage. At 12 weeks of age, the c-row of vibrissae was plucked under light isoflurane anesthesia in a manner that prevented follicle damage. After plucking, the mice were separated into three groups. The first, referred to as "deprived," consisted of mice that were simply returned to their original home cage. Mice in the "minimally enriched" group were returned to their home cage but also had a novel item added, stimulating vibrissae use but not necessarily having the broad effects of multiple levels of enrichment. The third group of mice, "deprived to enriched," were placed in an enriched environment after vibrissae removal. The enriched environment consisted of a large, bilevel cage $(61 \times 60 \times 60 \mathrm{~cm})$ that contained items such as tunnels, wire mesh, and running wheels, and were housed six to eight mice to a cage. Finally, the effect of prolonged enrichment was investigated through a fourth "enriched" group, which consisted of mice that were housed in an enriched environment before and after vibrissae plucking.

Mice remained in their assigned housing conditions for $6 \mathrm{~h}, 48 \mathrm{~h}, 7 \mathrm{~d}$, or $14 \mathrm{~d}$ ( $n=6$ per group per duration) after vibrissae plucking. Additionally, control mice that underwent the same protocols as above without vibrissae plucking were examined.

After the designated time had elapsed, the mice were prepared for histochemical staining of vesicular zinc by intraperitoneal administration of sodium selenite (15 mg/kg, i.p.; Sigma). After 1 h, mice were killed using an overdose of sodium pentobarbital. Brains were bisected after extraction and the cortices gently removed. To visualize the barrel cortex in a single plane, cortices were flattened between glass slides and then rapidly frozen. Sections were cut on a cryostat to $20 \mu \mathrm{m}$.

The Danscher autometallographic staining method was used to selectively visualize the histochemically reactive zinc present within vesicles of axon terminals (Danscher, 1982). To do this, sections were thawed at room temperature, fixed in $95 \%$ ethanol for $15 \mathrm{~min}$, hydrated in a descending series of ethanol and water (75\%, $2 \mathrm{~min} ; 50 \%, 2 \mathrm{~min}$; distilled water $3 \times 2 \mathrm{~min}$ ), and then dipped in a $0.5 \%$ gelatin solution. Sections were placed in the developing solution consisting of $50 \%$ gum arabic ( $100 \mathrm{ml}), 2.0 \mathrm{M}$ sodium citrate buffer $(25 \mathrm{ml}), 0.5 \mathrm{M}$ hydroquinone (30 $\mathrm{ml}), 37 \mathrm{~mm}$ silver lactate $(30 \mathrm{ml})$, and $20 \mathrm{ml}$ of distilled water for $1.5 \mathrm{~h}$. After development, sections were rinsed under warm tap water $\left(37^{\circ} \mathrm{C}, 10\right.$ $\mathrm{min})$ and distilled water $(2 \times 2 \mathrm{~min})$. Development was halted by immersing the sections in sodium thiosulfate $(5 \%, 12 \mathrm{~min})$. After rinsing (distilled water, $3 \times 2 \mathrm{~min}$ ), sections were fixed and dehydrated in an ascending series of ethanol (70\%, $30 \mathrm{~min}$; 95\%, $5 \mathrm{~min}$; 100\%, $10 \mathrm{~min}$ ), cleared in xylene (10 min), and coverslipped using Permount.

Images were captured digitally (Qimaging monobit 10-bit camera; Zeiss Axioskop2 microscope) and stored on an Apple iMac Intel Core Duo computer using Openlab imaging software (version 4.0.4, Improvision). All images were captured at a 256 grayscale.

Densitometric analysis was conducted using Openlab. Four to five adjacent sections that represented the majority of the posterior medial barrel subfield were captured. To control for inherent, between-animal differences in staining intensity, a ratio was calculated that compared the mean grayscale values for the deprived row (c-row; numerator) and the adjacent nondeprived rows (b- and d-row; denominator). The staining intensity between the c-row and b/d-rows of barrels is not consistent in unmanipulated mice (Brown and Dyck, 2002), and to ensure that our environmental manipulations did not alter this basal difference and our results, we also calculated a ratio value for control mice that were exposed to our environmental manipulations but did not undergo vibrissae plucking. We then calculated a relative percentage difference in staining intensity by comparing the average ratio obtained in the experimental group with that obtained in the corresponding control group.

All image quantifications were conducted while the investigator was blind to the experimental conditions. The data were submitted to a $4 \times 4$ (group $\times$ time point) factorial ANOVA. Significant main effects and interactions were followed up using the Tukey test.

\section{Results}

Consistent with previous findings, the removal of vibrissae resulted in robust increases in vesicular zinc within the corresponding barrels (Czupryn and Skangiel-Kramska, 2001b; Brown and Dyck, 2002). Representative images can be found in Figure 2, while Figure 3 displays the quantified data.

The main effects of group $\left(F_{(3,176)}=24.2, p<0.001\right)$ and time point $\left(F_{(3,176)}=20.1, p<0.001\right)$ were significant, as was the interaction $\left(F_{(9,176)}=4.26, p<0.001\right)$. The percentage increase in vesicular zinc staining was lowest in the deprived group at all time points examined. At the $6 \mathrm{~h}$ survival time point, a significantly greater increase in vesicular zinc staining was observed in the minimally enriched group compared with the deprived group, whereas at all other time points, no significant differences were found between these two groups. Although the increase in staining intensity of the deprived to enriched group was relatively low at the 6 and $48 \mathrm{~h}$ time points, at $7 \mathrm{~d}$, a significantly greater increase in vesicular zinc staining was observed after vibrissae removal, compared with the deprived and minimally enriched groups. At $14 \mathrm{~d}$, the deprived to enriched group had a significantly greater increase only when compared with the deprived group.

The enriched group consistently had an augmented increase in vesicular zinc staining of the barrels deprived of sensory input. At the $6 \mathrm{~h}$ time point, the increase was significantly different from the deprived to enriched group only, whereas at all other time 
Deprived
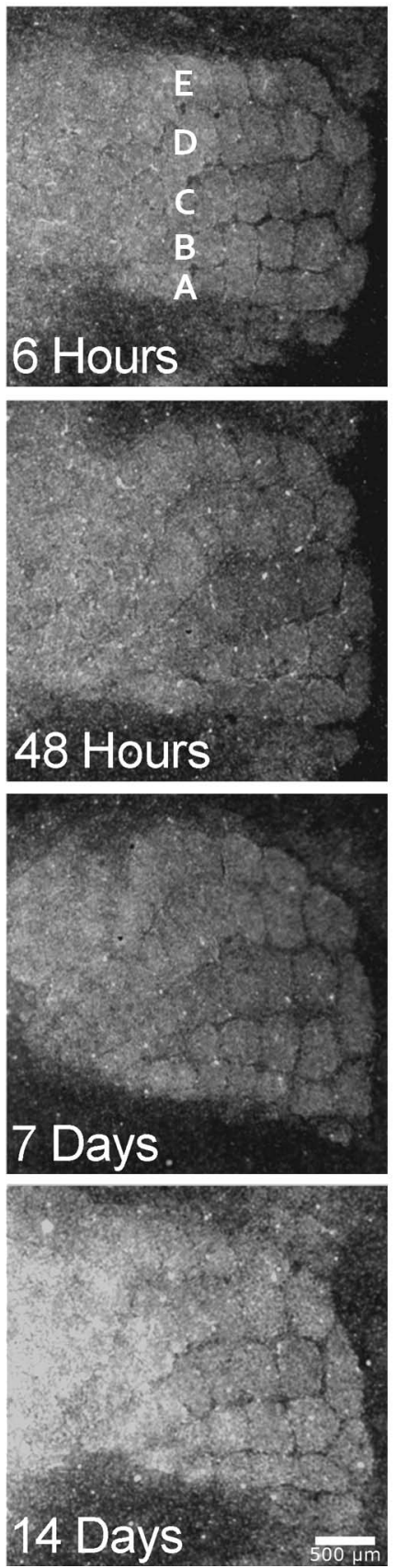

Minimally Enriched
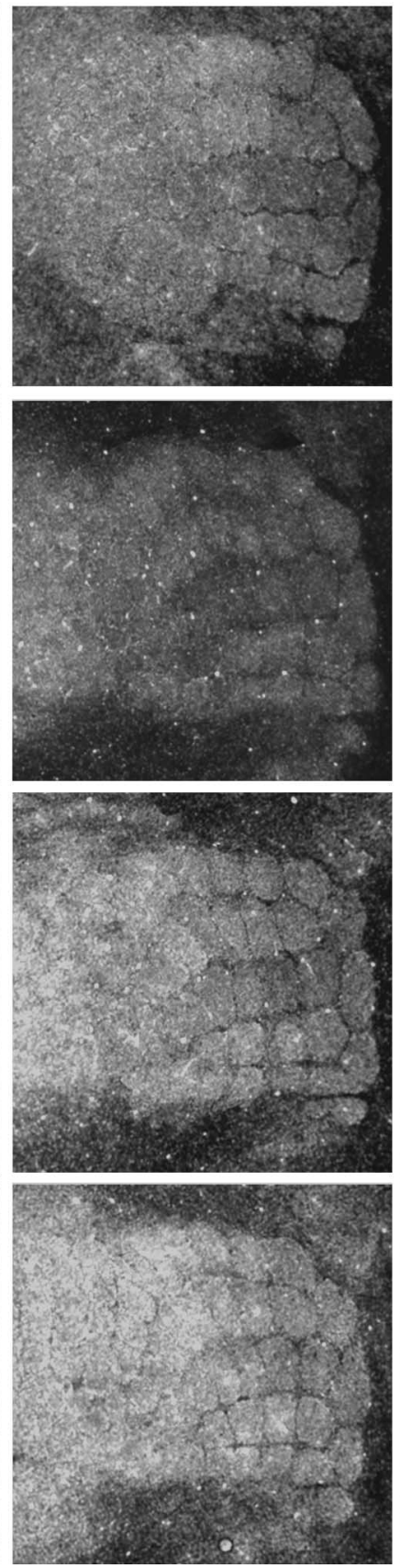

\section{Deprived to} Enriched
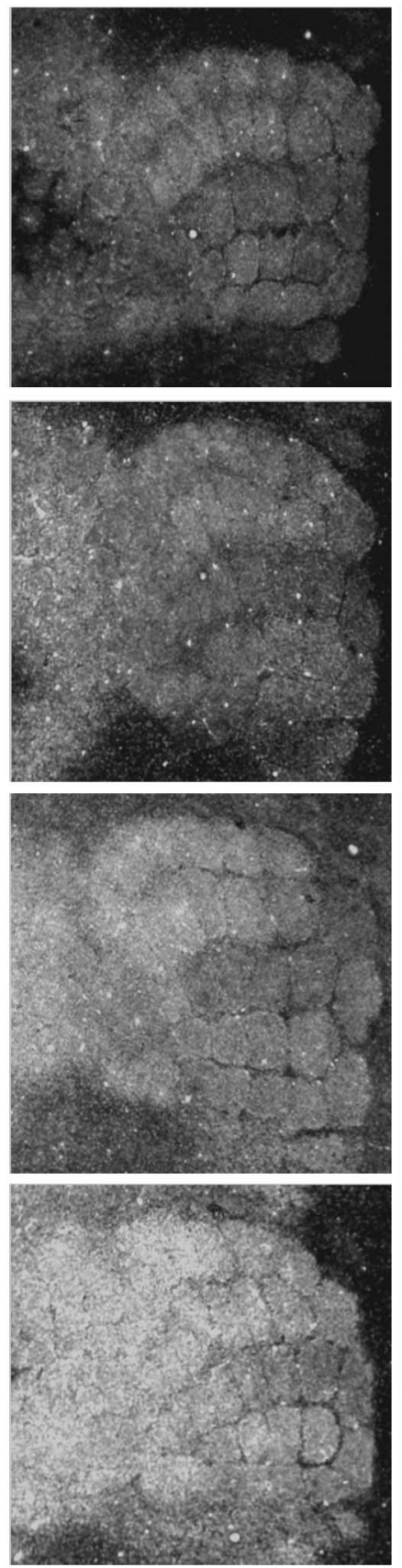
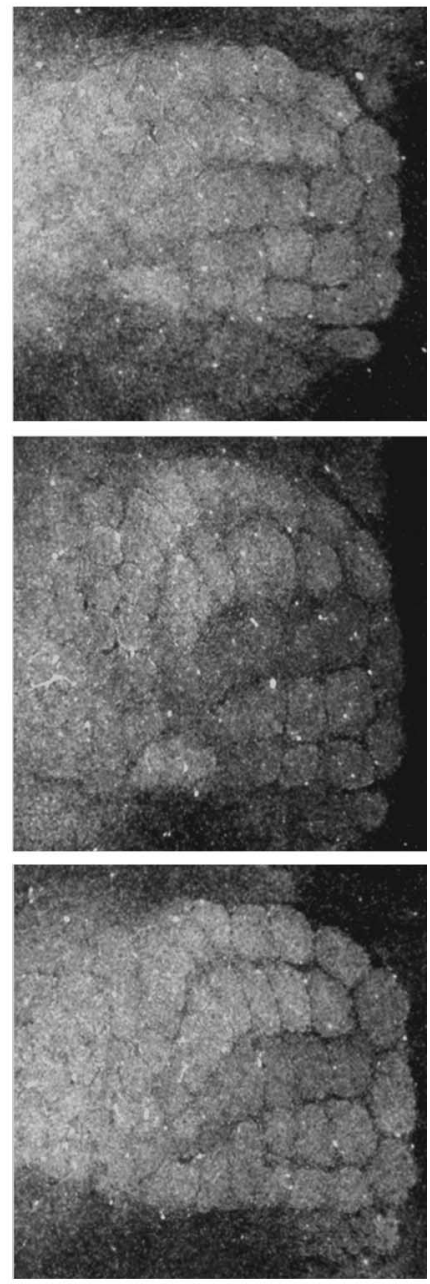

\section{Enriched}

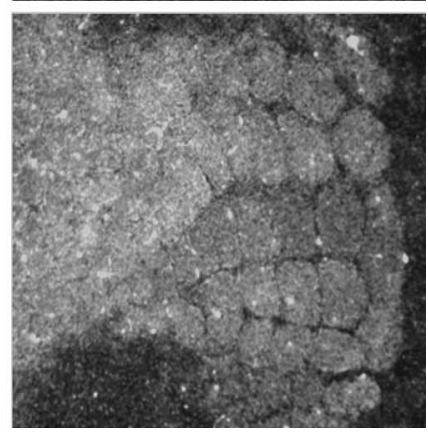

Figure 2. Experience-dependent regulation of vesicular zinc. The somatosensory cortex is well defined by vesicular zinc staining, and the five rows (A-E) of barrels corresponding to the vibrissae are easily resolved. Although an increase in zinc staining was observed in the c-row of all mice, the increases were noticeably augmented in the mice exposed to an enriched environment. Scale bar, $500 \mu \mathrm{m}$.

points examined, a significantly greater change was observed when comparing the enriched group with both the deprived and minimally enriched groups.

\section{Discussion}

In the present study, we explored whether manipulating the environmental housing conditions of mice would affect the regulation of vesicular zinc occurring concurrently with cortical plasticity induced by vibrissae plucking. We found that, in a manner dependent on the duration and degree of exposure, environmental enrichment led to a greater zincergic response to vibrissae plucking. Mice housed without enrichment were found to con- sistently have the lowest increase in vesicular zinc after vibrissae plucking. Conversely, mice housed in enriched conditions for the duration of the experiment had the greatest increase in vesicular zinc at all time points examined. Intermediate increases in vesicular zinc were observed with the minimal enrichment and deprived to enriched paradigms. Thus, we observe an increase in vesicular zinc within barrels deprived of sensory input that is correlated with the level of enrichment.

We found it interesting that minimal enrichment resulted in an immediate but brief amplification of the zincergic response to vibrissae plucking, whereas the deprived to enriched housing 


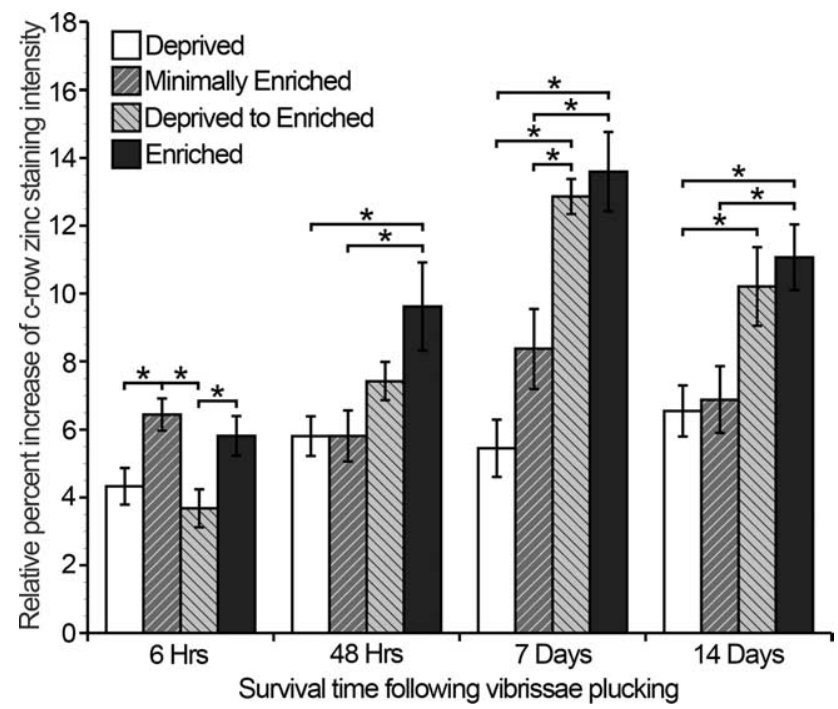

Figure 3. Quantification of changes in vesicular zinc staining in the barrel cortex after the plucking of the c-row of vibrissae in mice housed in enriched conditions. Percentage change in zinc staining reflects the difference in staining intensity ratio values between experimental (plucked) and control animals. Minimal enrichment had a small, but significant effect on the change in zinc staining only at the $6 \mathrm{~h}$ time point. Mice housed in the enriched environment consistently had a significantly greater change in zinc staining compared with the deprived condition at all time points. Exposure to an enriched environment after vibrissae removal (deprived to enriched) resulted in an augmented change compared with the deprived group, but only at the later time points. Error bars are \pm SEM, ${ }^{*} p<0.05$.

condition resulted in a large increase in vesicular zinc levels only at the longer survival time points. It is likely that these responses are reflective of the effectiveness of environmental manipulations. Minimal enrichment promotes activity in the short term, but it decreases rapidly as the uniqueness of the novel item fades. In the deprived to enriched condition, the exposure of the mice to a new environment with strange cohorts may initially induce a stress response that reduces activity and prevents immediate enrichment-related cortical changes from occurring. Alternatively, it is known from other studies that the effects of enrichment are not immediate, taking between $12 \mathrm{~h}$ and $4 \mathrm{~d}$ of exposure to enrichment to observe any consequences (Ferchmin et al., 1970; Diamond et al., 1976; Henderson, 1976; Rema et al., 2006).

Environmental conditions that promote activity through physical, mental, and social stimulation lead to a number of alterations in the morphology and physiology of the cortex (Nithianantharajah and Hannan, 2006). Many of the changes involve factors that are important for cortical plasticity, such as alterations in NMDA receptor subunit expression (Tang et al., 2001), production of growth factors like brain-derived neurotrophic factor (BDNF) (Ickes et al., 2000), and the expression of other genes and gene products associated with synaptic plasticity (Rampon et al., 2000; McNair et al., 2007). These alterations in turn affect synaptic plasticity, because LTP/LTD expression is modified by enrichment (Li et al., 2006) and, presumably, cortical plasticity. Together with previous research that supports the contribution of zinc to cortical synaptic plasticity, our results indicate that the regulation of zinc levels provides another level of control, and that it is enhanced with enrichment.

A major consideration is whether the increase in vesicular zinc is integral to the changes in cortical plasticity caused by environmental enrichment, or whether our observations are epiphenomenonal. We argue for the former based on the following arguments. First, the large increases in vesicular zinc levels we observed were not passively caused by enrichment; instead, they also required modifications of sensory input in the form of vibrissae removal. Further support comes from previous studies showing that vesicular zinc levels rapidly increased or decreased in the barrel cortex depending on whether input from the vibrissae was reduced by plucking or enhanced by stimulation, respectively (Czupryn and Skangiel-Kramska, 2001a; Brown and Dyck, 2002, 2005). We take this to be indicative of a role for zinc in experience-dependent plasticity. Alternatively, it could be presumed that these modulations simply reflect changes in the activity of neurons within the barrels of interest. For example, after silencing vibrissal input, synaptic activity within the corresponding barrels would decrease, leading to the retention of zinc within presynaptic vesicles. Such a process would also require that uptake levels remained constant and independent of activity. However, in the context of the present results, it would imply that zincergic neurons in the enriched brain were less able to maintain zinc homeostasis. Although this has not been specifically examined, in light of the increased capabilities that enrichment confers on cortical function, it seems extremely unlikely.

Instead, we argue that it is more likely that the neuromodulatory effects of zinc are enhanced by enrichment and thereby exert a greater influence on the mechanisms of cortical plasticity. For instance, zinc can modulate NMDA and AMPA receptor activity (Peters et al., 1987; Westbrook and Mayer, 1987; Mott et al., 2008), alter levels of BDNF mRNA (Nowak et al., 2004), modify postsynaptic proteins (Baron et al., 2006), and directly influence the induction of LTP/LTD (Xie and Smart, 1994; Izumi et al., 2006; Huang et al., 2008). These processes have all been shown to be modified by enriched environments. By modifying them, changes in zinc signaling could influence the induction and expression of cortical plasticity in the enriched brain.

The most likely mechanism by which zinc modulates plasticity is through its actions on NMDA receptors. Zinc inhibits the activity of NMDA receptors in a concentration-dependent manner (Christine and Choi, 1990; Chen et al., 1997; Paoletti et al., 1997), and, as such, an increase in vesicular zinc levels would result in a greater inhibition of NMDA receptors. Because the dynamics of NMDA receptor activation are crucial components of many types of cortical plasticity (for review, see Buonomano and Merzenich, 1998; Malenka and Bear, 2004), reductions in the relative activity of these receptors would result in the weakening of synaptic connectivity associated with deprived inputs, and allowing adjacent inputs to encroach on them, thereby facilitating the reorganization of cortical circuitry in the enriched animal.

Based on our results, future studies should be directed at determining the precise role of vesicular zinc on the functioning of the enriched brain, especially in regard to cortical plasticity. Studies using the ZnT3 knock-out mouse, a transgenic line lacking the transporter responsible for packaging zinc into synaptic vesicles and, therefore, lacking vesicular zinc (Cole et al., 1999), would be extremely informative. Environmental enrichment of these mice might reveal those components of plasticity that are enhanced by enrichment and are mediated by zinc. For example, changes in synaptic plasticity, in the form of LTP/LTD, experiencedependent plasticity, in the form of map alterations after sensory manipulations, or learning, in the form of behavioral tests, after exposure to environmental enrichment in the ZnT3 knock-out mouse would be indicative of the integral involvement of vesicular zinc in enrichment-induced cortical modifications.

Cortical plasticity is dictated by experiential factors that drive neuronal activity, maintaining and changing the circuitry therein. In the present study, we showed that the regulation of 
vesicular zinc changes significantly when the complexity of experiential features is manipulated. Although much remains to be ascertained regarding the functional significance of these alterations, given the multitude of effects zinc can have on neurotransmission and synaptic plasticity, it is likely that active modulations of zinc levels provide an additional mechanism by which cortical plasticity can be induced.

\section{References}

Abraham WC, Logan B, Greenwood JM, Dragunow M (2002) Induction and experience-dependent consolidation of stable long-term potentiation lasting months in the hippocampus. J Neurosci 22:9626-9634.

Artola A, von Frijtag JC, Fermont PC, Gispen WH, Schrama LH, Kamal A, Spruijt BM (2006) Long-lasting modulation of the induction of LTD and LTP in rat hippocampal CA1 by behavioural stress and environmental enrichment. Eur J Neurosci 23:261-272.

Assaf SY, Chung SH (1984) Release of endogenous Zn2+ from brain tissue during activity. Nature 308:734-736.

Baron MK, Boeckers TM, Vaida B, Faham S, Gingery M, Sawaya MR, Salyer D, Gundelfinger ED, Bowie JU (2006) An architectural framework that may lie at the core of the postsynaptic density. Science 311:531-535.

Beaulieu C, Dyck R, Cynader M (1992) Enrichment of glutamate in zinccontaining terminals of the cat visual cortex. Neuroreport 3:861-864.

Bender KJ, Allen CB, Bender VA, Feldman DE (2006) Synaptic basis for whisker deprivation-induced synaptic depression in rat somatosensory cortex. J Neurosci 26:4155-4165.

Brown CE, Dyck RH (2002) Rapid, experience-dependent changes in levels of synaptic zinc in primary somatosensory cortex of the adult mouse. J Neurosci 22:2617-2625.

Brown CE, Dyck RH (2005) Modulation of synaptic zinc in barrel cortex by whisker stimulation. Neuroscience 134:355-359.

Buonomano DV, Merzenich MM (1998) Cortical plasticity: from synapses to maps. Annu Rev Neurosci 21:149-186.

Chen N, Moshaver A, Raymond LA (1997) Differential sensitivity of recombinant N-methyl-D-aspartate receptor subtypes to zinc inhibition. Mol Pharmacol 51:1015-1023.

Christine CW, Choi DW (1990) Effect of zinc on NMDA receptor-mediated channel currents in cortical neurons. J Neurosci 10:108-116.

Cole TB, Wenzel HJ, Kafer KE, Schwartzkroin PA, Palmiter RD (1999) Elimination of zinc from synaptic vesicles in the intact mouse brain by disruption of the ZnT3 gene. Proc Natl Acad Sci U S A 96:1716-1721.

Czupryn A, Skangiel-Kramska J (2001a) Deprivation and denervation differentially affect zinc-containing circuitries in the barrel cortex of mice. Brain Res Bull 55:287-295.

Czupryn A, Skangiel-Kramska J (2001b) Differential response of synaptic zinc levels to sensory deprivation in the barrel cortex of young and adult mice. Exp Brain Res 141:567-572.

Danscher G (1982) Exogenous selenium in the brain. A histochemical technique for light and electron microscopical localization of catalytic selenium bonds. Histochemistry 76:281-293.

Diamond MC, Ingham CA, Johnson RE, Bennett EL, Rosenzweig MR (1976) Effects of environment on morphology of rat cerebral cortex and hippocampus. J Neurobiol 7:75-85.

Ferchmin PA, Eterovic VA, Caputto R (1970) Studies of brain weight and RNA content after short periods of exposure to environmental complexity. Brain Res 20:49-57.

Foster TC, Dumas TC (2001) Mechanism for increased hippocampal synaptic strength following differential experience. J Neurophysiol 85:1377-1383.

Foster TC, Gagne J, Massicotte G (1996) Mechanism of altered synaptic strength due to experience: relation to long-term potentiation. Brain Res 736:243-250.

Frederickson CJ (1989) Neurobiology of zinc and zinc-containing neurons. Int Rev Neurobiol 31:145-238.

Glazewski S, Fox K (1996) Time course of experience-dependent synaptic potentiation and depression in barrel cortex of adolescent rats. J Neurophysiol 75:1714-1729.

Henderson ND (1976) Short exposures to enriched environments can increase genetic variability of behavior in mice. Dev Psychobiol 9:549-553.

Howell GA, Welch MG, Frederickson CJ (1984) Stimulation-induced uptake and release of zinc in hippocampal slices. Nature 308:736-738.

Huang YZ, Pan E, Xiong ZQ, McNamara JO (2008) Zinc-mediated transactivation of TrkB potentiates the hippocampal mossy fiber-CA3 pyramid synapse. Neuron 57:546-558.

Ickes BR, Pham TM, Sanders LA, Albeck DS, Mohammed AH, Granholm AC (2000) Long-term environmental enrichment leads to regional increases in neurotrophin levels in rat brain. Exp Neurol 164:45-52.

Irvine GI, Logan B, Eckert M, Abraham WC (2006) Enriched environment exposure regulates excitability, synaptic transmission, and LTP in the dentate gyrus of freely moving rats. Hippocampus 16:149-160.

Izumi Y, Auberson YP, Zorumski CF (2006) Zinc modulates bidirectional hippocampal plasticity by effects on NMDA receptors. J Neurosci 26:7181-7188.

Kempermann G, Kuhn HG, Gage FH (1997) More hippocampal neurons in adult mice living in an enriched environment. Nature 386:493-495.

Leggio MG, Mandolesi L, Federico F, Spirito F, Ricci B, Gelfo F, Petrosini L (2005) Environmental enrichment promotes improved spatial abilities and enhanced dendritic growth in the rat. Behav Brain Res 163:78-90.

Li S, Tian X, Hartley DM, Feig LA (2006) The environment versus genetics in controlling the contribution of MAP kinases to synaptic plasticity. Curr Biol 16:2303-2313.

Li Y, Hough CJ, Frederickson CJ, Sarvey JM (2001) Induction of mossy fiber $\rightarrow$ Ca3 long-term potentiation requires translocation of synaptically released Zn2+. J Neurosci 21:8015-8025.

Malenka RC, Bear MF (2004) LTP and LTD: an embarrassment of riches. Neuron 44:5-21.

McNair K, Broad J, Riedel G, Davies CH, Cobb SR (2007) Global changes in the hippocampal proteome following exposure to an enriched environment. Neuroscience 145:413-422.

Mott DD, Benveniste M, Dingledine RJ (2008) pH-dependent inhibition of kainate receptors by zinc. J Neurosci 28:1659-1671.

Nithianantharajah J, Hannan AJ (2006) Enriched environments, experience-dependent plasticity and disorders of the nervous system. Nat Rev 7:697-709.

Nowak G, Legutko B, Szewczyk B, Papp M, Sanak M, Pilc A (2004) Zinc treatment induces cortical brain-derived neurotrophic factor gene expression. Eur J Pharmacol 492:57-59.

Paoletti P, Ascher P, Neyton J (1997) High-affinity zinc inhibition of NMDA NR1-NR2A receptors. J Neurosci 17:5711-5725.

Peters S, Koh J, Choi DW (1987) Zinc selectively blocks the action of N-methyl-D-aspartate on cortical neurons. Science 236:589-593.

Rampon C, Jiang CH, Dong H, Tang YP, Lockhart DJ, Schultz PG, Tsien JZ, $\mathrm{Hu} \mathrm{Y} \mathrm{(2000)} \mathrm{Effects} \mathrm{of} \mathrm{environmental} \mathrm{enrichment} \mathrm{on} \mathrm{gene} \mathrm{expression} \mathrm{in}$ the brain. Proc Natl Acad Sci U S A 97:12880-12884.

Rema V, Armstrong-James M, Jenkinson N, Ebner FF (2006) Short exposure to an enriched environment accelerates plasticity in the barrel cortex of adult rats. Neuroscience 140:659-672.

Sirevaag AM, Greenough WT (1988) A multivariate statistical summary of synaptic plasticity measures in rats exposed to complex, social and individual environments. Brain Res 441:386-392.

Tang YP, Wang H, Feng R, Kyin M, Tsien JZ (2001) Differential effects of enrichment on learning and memory function in NR2B transgenic mice. Neuropharmacology 41:779-790.

Venable N, Pinto-Hamuy T, Arraztoa JA, Contador MT, Chellew A, Perán C, Valenzuela X (1988) Greater efficacy of preweaning than postweaning environmental enrichment on maze learning in adult rats. Behav Brain Res 31:89-92.

Westbrook GL, Mayer ML (1987) Micromolar concentrations of Zn2+ antagonize NMDA and GABA responses of hippocampal neurons. Nature 328:640-643.

Xie X, Smart TG (1994) Modulation of long-term potentiation in rat hippocampal pyramidal neurons by zinc. Pflugers Arch 427:481-486. 